\title{
On the Quasi-Asymptotic Expansion of the Causal Fundamental Solution of Hyperbolic Operators and Systems
}

\author{
P. WAGNER
}

\begin{abstract}
Explicit formulae for the quasi-asymptotic expansion (in the sense of V.S. Vladimirov) of the causal fundamental solution of hyperbolic partial differential operators resp. systems with constant coefficients are given. The method is applied to some concrete examples.

Key words: Quasi-asymptotic expansion, fundamental solutions, linear partial differential operators, hyperbolic operators

AMS subject classification: $46 \mathrm{~F}, 35 \mathrm{~L}$
\end{abstract}

\section{INTRODUCTION}

In the beautiful monograph [9], V. S. Vladimirov, Yu. N. Drozhzhinov and B. I. Zav'yalov presented a systematic treatment of multidimensional Tauberian theorems for distributions. Among other applications, they employ this theory to determine the quasi-asymptotic of causal fundamental solutions $E$ of linear hyperbolic partial differential operators and of linear passive systems (cf. [9, Ch. IV]). As a general rule, a supplemental condition has to be presumed, in order to validate a Tauberian theoremthat is, to draw a conclusion on the behaviour of a distribution at infinity from that of its Laplace transform at zero. In the applications mentioned, this rôle is played by the fact that the argument of the Laplace transform of $E$ is bounded (see $[9, \S 4.4$; Lemma 1, $\$ 11.2$; Proof of Theorem 1, $\$ 12.3]$ ). It is the purpose of this paper to describe a different approach which also allows to calculate the quasi-asymptotic and even the quasi-asymptotic expansion of causal fundamental solutions. Instead of relying on the principle of bounded argument, the present method is based on an estimate for polynomials given by L. Hörmander.

The notion of a quasi-asymptotic expansion with respect to an open convex cone $\Gamma$ in $\mathbf{A}^{n}$ is understood here in the following sense. If $\left\{\lambda_{n}\right\}_{n \geq 0}, \lambda_{0}<\lambda_{1}<\ldots$, is a sequence of real numbers and $T_{j}$ are distributions in $S^{\prime}(\Gamma)$ (necessarily homogeneous of the degree $-\lambda_{j}$ ), then the notation

$$
E(k x) \sim \sum_{j=0}^{\infty} k^{-\lambda_{j}} T_{j}(x), k \rightarrow \infty
$$

will mean that

$$
\forall J \in \mathbf{N}: \lim _{k \rightarrow \infty} k^{\lambda},\left[E(k x)-\sum_{j=0}^{J} k^{-\lambda_{j}} T_{j}(x)\right]=0 \text { in } S^{\prime}(\Gamma)
$$

(comp. $[9, \S 10]$ for the one-dimensional case). Let us observe that, in general, this series fails to converge in $S^{\prime}(\Gamma)$. 


\section{HYPERBOLIC OPERATORS}

A linear partial differential operator (with constant coefficients) $P(\partial)=\sum_{j=1}^{m} P_{j}(\partial)$, $P_{j}$ homogeneous of degree $j, P_{l} \neq \equiv 0, P_{m} \not \equiv 0$, is called hyperbolic with respect to the direction $N \in \mathbf{R}^{n} \backslash\{0\}$ if $P_{m}(N) \neq 0$ and $P(-i x+\tau N) \neq 0$ for all $x \in \mathbf{R}^{\mathbf{n}}$ and $\tau>\tau_{0}$ (cf. $\left[3\right.$, Def. 5.4.1]). We shall assume that $\tau_{0}=0$, which amounts to considering the shifted operator $P\left(\partial+\tau_{0} N\right)$ instead of $P(\partial)$. By Theorem 5.5 .5 of $[3], P(\partial)$ is hyperbolic with respect to all directions in an open convex cone $C:=\Gamma(P, N)$. A closer inspection of the proof of this theorem shows that in our case (that is, $\tau_{0}=0$ )

$$
P(-i z) \neq 0 \text { for } z \in T^{C}:=\mathrm{R}^{n}+i C \text {. }
$$

There exists exactly one "causal" fundamental solution $E$ of $P(\partial)$ having its support in $\Gamma:=C^{*}$ (cf. $\left[3\right.$, Theorem 5.6.1]) and, furthermore, $E \in S^{\prime}(\Gamma)$, see $[8, \S 13.2]$. Since

$$
P_{l}(-i z)=\lim _{k \rightarrow \infty} k^{l} P(-i z / k),
$$

the polynomial $P_{l}$ also satisfies $P_{l}(-i z) \neq 0$ for $z \in T^{C}$. This follows from the fact that, for fixed $z \in T^{c}$ and $\zeta \in C^{n}$ with $P_{l}(\zeta) \neq 0$, the number of zeros of the non-zero polynomial $P_{l}(-i z+\lambda s)$ in $\lambda$ within a bounded region $G$ in the complex plane can be expressed by the complex line integral

$$
\int_{\partial G} \frac{d}{d \lambda}\left(\log P_{l}(-i z+\lambda \zeta)\right) \frac{d \lambda}{2 \pi i}
$$

and hence must vanish if $\{-i z+\lambda \zeta: \lambda \in G\} \subset T^{C}$ (also compare the proof of Theorem 5.5.2 in [3]). Therefore, the homogeneous operator $P_{l}(\partial)$ and its powers are hyperbolic with respect to $C$.

Proposition 1. Let $P(\partial)=\sum_{j=1}^{m} P_{j}(\partial)$ and $\Gamma$ be as above and denote the causal fundamental solutions of $P(\partial)$ and of $P_{l}(\partial)^{i}$ by $E$ and $E_{l, i}$, respectively. Then $E$ has the following quasi-asymptotic expansion in $S^{\prime}(\Gamma)$ for $k \rightarrow \infty$ :

$$
E(k x) \sim \sum_{r=0}^{\infty} k^{l-n-r} T_{r}(x)
$$

wherein $T_{r}$ are homogeneous distributions of the degree $l-n-r$ given by

$$
T_{r}=\sum_{\alpha_{1}+2 \alpha_{2}+\cdots+(m-1) \alpha_{m-1}=r}\left(\begin{array}{c}
|\alpha| \\
\alpha
\end{array}\right)(-1)^{|\alpha|} \prod_{j=1}^{m-1} P_{l+j}(\partial)^{\alpha,} E_{l, 1+|\alpha|}
$$

PROOF: By the Laplace transformation, the convolution algebra $S^{\prime}(\Gamma)$ is isomorphically mapped onto the space $H(C)$ of the functions $f(z)$ which are holomorphic on $T^{C}$ and which fulfill an estimate of the form

$$
\exists \beta, \gamma, c>0:|f(z)| \leq c\left(1+|z|^{2}\right)^{\beta} \Delta_{C}(y)^{-\gamma} .
$$


Herein $z=x+i y$ and

$$
\Delta_{C}(y):=\operatorname{dist}(y, \partial C)=\min \{|y-u|: u \in \partial C\}
$$

(see $[8, \S 12],[9, \S 2.5])$. Therefore, the validity of the quasi-asymptotic expansion in $S^{\prime}(\Gamma)$ which is stated in the proposition is equivalent to that of the following quasiasymptotic expansion in $H(C)$ as $t \searrow 0$ :

$$
\begin{aligned}
P(-i z t)^{-1} \sim & -\sum_{r=0}^{\infty} t^{r-1} \sum_{a_{1}+2 \alpha_{2}+\cdots+(m-1) \alpha_{m-1}=r}\left(\begin{array}{c}
|\alpha| \\
\alpha
\end{array}\right) \frac{\prod_{j=1}^{m-1} P_{1+j}(-i z)^{\alpha}}{\left(-P_{l}(-i z)\right)^{|\alpha|+1}} \\
& =: \sum_{r=0}^{\infty} t^{r-1} Q_{r}(z) .
\end{aligned}
$$

For fixed $z \in T^{C}$, this series simply is the Laurent expansion around zero of the meromorphic function

$$
g(t):=P(-i z t)^{-1}=\left(\sum_{j=1}^{m} P_{j}(-i z) t^{j}\right)^{-1}
$$

in the complex $t$ - plane and hence is even convergent for $0<|t|<\epsilon(z)$. In order to show the asymptotic convergence in $H(C)$ for $t \searrow 0$, we have to verify instead that

$$
\lim _{t \backslash 0} t^{t-R}\left[P(-i z t)^{-1}-\sum_{r=0}^{R} t^{r-1} Q_{r}(z)\right]=0 \text { in } H(C)
$$

for $R \in \mathrm{N}$. Expanding the differentiable function $t^{\prime} g(t)$ in a power series around zero and representing the remainder by an integral we obtain for $t>0$

$$
t^{\prime} g(t)-\sum_{r=0}^{R} t^{r} Q_{r}(z)=\frac{1}{R !} \int_{0}^{t}(t-s)^{R}\left(\frac{d}{d s}\right)^{R+1}\left(s^{\prime} g(s)\right) d s
$$

and hence

$$
\begin{aligned}
\left|t^{i-R}\left[P(-i z t)^{-1}-\sum_{r=0}^{R} t^{r-1} Q_{r}(z)\right]\right| & \leq \frac{1}{t^{R} R !} \int_{0}^{t}(t-s)^{R}\left|\left(\frac{d}{d s}\right)^{R+1}\left(\frac{s^{t}}{P(-i z s)}\right)\right| d s \\
& \leq \frac{1}{R !} \int_{0}^{t}\left|\left(\frac{d}{d s}\right)^{R+1}\left(\frac{s^{t}}{P(-i z s)}\right)\right| d s .
\end{aligned}
$$

Therefore, it remains to be shown that

$$
\lim _{t \backslash 0} \max _{x \in T^{c}}\left[\left(1+|z|^{2}\right)^{-\theta} \Delta_{C}(y)^{\gamma} \int_{0}^{t}\left|\left(\frac{d}{d s}\right)^{R+1}\left(\frac{s^{l}}{P(-i z s)}\right)\right| d s\right]=0
$$


for some $\beta, \gamma>0$. Since

$$
\left(\frac{d}{d s}\right)^{R+1}\left(\frac{s^{l}}{P(-i z s)}\right)=\left(\frac{s^{l} \cdot}{P(-i z s)}\right)^{R+2} P_{1}(z, s)
$$

for some polynomial $P_{1}(z, s)$, we are allowed to replace the integral in the above limit by the following one:

$$
\int_{0}^{t}\left|\left(\frac{s^{t}}{P(-i z s)}\right)^{R+2}\right| d s=2 \int_{0}^{\sqrt{i}}\left|\left(\frac{\sigma^{2 l}}{P\left(-i z \sigma^{2}\right)}\right)^{R+2}\right| \sigma d \sigma .
$$

If we regard $\sigma^{-21} P\left(-i z \sigma^{2}\right)$ as a polynomial $Q(x, y, \sigma)$ in the $2 n+1$ real variables $x, y, \sigma$, then the set $N$ of its real zeros fulfills

$$
N \subset \mathbf{R}_{x}^{n} \times\left(\mathbf{R}_{y}^{n} \backslash C\right) \times \mathbf{R}_{\sigma}^{1}
$$

and hence, if "dist" denotes the Euclidean distance in $\mathbf{R}^{2 n+1}$, we have

$$
\operatorname{dist}((x, y, \sigma) ; N) \geq \Delta_{C}(y) \text { for } z=x+i y \in T^{C} \text { and } \sigma \in \mathbf{R} .
$$

By Lemma 2 in $[2]$, this estimate implies

$$
\exists c, \mu^{\prime}, \mu^{\prime \prime}>0: \forall z \in T^{C}: \forall \sigma \text { with } 0<\sigma<1:\left|\frac{\sigma^{2 l}}{P\left(-i z \sigma^{2}\right)}\right| \leq c\left(1+|z|^{2}\right)^{\mu^{\prime}} \Delta_{C}(y)^{-\mu^{\prime \prime}},
$$

and thus the required limit in $H(C)$ follows.

REMARKS. 1) The quasi-asymptotic expansion of $E$ for $k \searrow 0$ can be derived in exactly the same way. Keeping the above notations we obtain for $k \searrow 0$ :

$$
E(k x) \sim \sum_{r=0}^{\infty} k^{m-n+r} S_{r}(x)
$$

wherein $S_{r}$ are homogeneous distributions of the degree $m-n+r$ given by

$$
S_{r}=\sum_{\substack{\alpha_{m-1}+2 \alpha_{m-2+\cdots+m \alpha_{0}=r} \\
\alpha_{i} \geq 0}}\left(\begin{array}{c}
|\alpha| \\
\alpha
\end{array}\right)(-1)^{|\alpha|} \prod_{j=0}^{m-1} P_{j}(\partial)^{\alpha_{j}} E_{m, 1+|\alpha|}
$$

This expansion, though generally divergent in $S^{\prime}(\Gamma)$, happens to converge in $D^{\prime}$, or, more precisely, in $B_{\infty}^{\text {loc }} \widetilde{P_{m}}$, cf. $[4$, Th. 12.5.3].

2) Proposition 1 can be generalized to also contain the case of a quasihomogeneous decomposition of $P(\partial)$ (compare $\left[10\right.$, p. 409]). Namely, if $a_{1}, \ldots, a_{n} \in N$ and if

$$
P_{j}\left(k^{a_{1}} x_{1}, \ldots, k^{a_{n}} x_{n}\right)=k^{j} P_{j},
$$


then

$$
E\left(k^{a_{1}} x_{1}, \ldots, k^{a_{n}} x_{n}\right) \sim \sum_{r=0}^{\infty} k^{l-|a|-r} T_{r}(x), \quad v k \rightarrow \infty,
$$

$T_{r}$ being given as in Prop. 1. This type of expansion is specially adapted to comparing the Timoshenko beam operator, viz.

$$
\left(\frac{1}{v_{1}^{2}} \partial_{t}^{2}-\partial_{x}^{2}\right)\left(\frac{1}{v_{2}^{2}} \partial_{t}^{2}-\partial_{x}^{2}\right)+\frac{1}{c^{2}} \partial_{t}^{2}
$$

with the ordinary beam operator $c^{-2} \partial_{t}^{2}+\partial_{x}^{4}$, which example will be treated in more detail in a forthcoming joint paper with $\mathrm{N}$. Ortner.

EXAMPLE 1 (cf. $[\mathbf{9}, \S 11.2])$. Let us apply the proposition to the Klein-Gordon operator in $n-1$ space dimensions

$$
P(\partial)=\square_{n}+c^{2}=\partial_{0}^{2}-\Delta_{n-1}+c^{2}, \Delta_{n-1}=\partial_{1}^{2}+\cdots+\partial_{n-1}^{2}, c>0 .
$$

In this case, $E$ is the value in $\zeta=1$ of the function

$$
\begin{aligned}
& h(\varsigma, c):=\frac{c^{n / 2}}{(2 \pi)^{n / 2-1}} \frac{s^{s-n / 2}}{\Gamma(\varsigma)(2 c)^{s}} J_{s-\frac{n}{2}}(c s), \\
& \qquad s=\left(x_{0}^{2}-x_{1}^{2}-\cdots-x_{n-1}^{2}\right)^{1 / 2}, \quad \operatorname{Re} \varsigma>\frac{n-1}{2},
\end{aligned}
$$

which, as a distribution-valued function, can holomorphically be continued to the whole complex 5 -plane (cf. [6, Ch. VI, §5], [5, p. 166]). Proposition 1 yields the following quasi-asymptotic expansion for $k \rightarrow \infty$ :

$$
E(k x) \sim-\sum_{j=0}^{\infty} \frac{k^{-n-2 j}}{\left(-c^{2}\right)^{j+1}} \square_{n}^{j} \delta
$$

EXAMPLE 2 (cf. $[9, \S 11.2]$ ). The so-called telegraph operator $\square_{n}-c^{2}, c>0$, assumes, after the shift $\partial_{0} \longrightarrow \partial_{0}+c$, the form $P(\partial)=\square_{n}+2 c \partial_{0}$, which operator satisfies the condition

$$
\forall z \in T^{C}: P(-i z) \neq 0 ; C=\left\{y: y_{0}^{2}>y_{1}^{2}+\cdots+y_{n-1}^{2}\right\} \text {. }
$$

The fundamental solution $E$ is given by $e^{-c x_{0}} h(1, i c) ; h$ being defined as before. Proposition 1 furnishes

$$
E(k x) \sim-\sum_{j=0}^{\infty} \frac{k^{1-n-j}}{(-2 c)^{j+1} j !} \square_{n}^{j} Y\left(x_{0}\right) x_{0}^{j} \otimes \delta_{x_{1}} \otimes \cdots \otimes \delta_{x_{n-1}},
$$

wherein $Y$ denotes Heaviside's function. 


\section{HYPERBOLIC SYSTEMS}

Let $Z(\partial)$ be a system of $N$ linear partial differential equations (with constant coefficients) containing $N$ unknown functions, i. e., $Z(\partial)$ is an $N \times N$-matrix of operators. Assume that $P(\partial):=\operatorname{det} Z(\partial)$ fulfills the requirements of Section 2-that is, $P(-i z) \neq 0$ for $z \in T^{C}$. The unique (again called causal) two-sided fundamental solution $E_{Z}$ of $Z(\partial)$ having its support in $\Gamma:=C^{*}$ is given by the formula $E_{z}=Z^{\mathrm{adj}}(\partial) E$, wherein $Z^{\mathrm{adj}}$ denotes the adjoint matrix to $Z$ and $E$ is the causal fundamental solution of $P(\partial)$ (cf. $[3,3.8]$ ). Let us decompose $Z^{\text {adi }}$ into its homogeneous components:

$$
Z^{\mathrm{adj}}=\sum_{\cdot=i_{1}}^{m_{1}} Z_{\bullet}^{\mathrm{adj}}, Z_{\cdot, i j}^{\mathrm{sdj}} \text { homogeneous of degree } s, 1 \leq i, j \leq N .
$$

Then we immediately obtain the quasi-asymptotic expansion of $E_{Z}$ from that of $E$ :

$$
E(k x) \sim \sum_{r=0}^{\infty} k^{l-n-r} T_{r}(x) \Longrightarrow E_{Z}(k x) \sim \sum_{r=l_{1}}^{\infty} k^{l-n-r} \sum_{\cdot=l_{1}}^{\min \left\{m_{1, r}\right\}} Z_{a}^{\text {sd } j}(\partial) T_{r-.}(x)
$$

EXAMPLE 3 (cf. $[9, \S 12.5])$. In the study of the motion of a rotating liquid, one is led to a system of partial differential equations represented by the following matrix:

$$
Z(\partial)=\left(\begin{array}{cccc}
\sigma \partial_{0} & \partial_{1} & \partial_{2} & \partial_{3} \\
\partial_{1} & \partial_{0} & \eta & 0 \\
\partial_{2} & -\eta & \partial_{0} & 0 \\
\partial_{3} & 0 & 0 & \partial_{0}
\end{array}\right), \quad \sigma, \eta>0
$$

An easy calculation furnishes

$$
P(\partial)=\operatorname{det} Z(\partial)=\partial_{0}^{2}\left(\sigma \partial_{0}^{2}-\Delta_{3}\right)+\eta^{2}\left(\sigma \partial_{0}^{2}-\partial_{3}^{2}\right)
$$

and thus, from Proposition 1, we obtain

$$
\begin{gathered}
E(k x) \sim \sum_{j=0}^{\infty} k^{-2-2 j} T_{2 j}=\sum_{j=0}^{\infty} k^{-2-2 j}\left(-\partial_{0}^{2}\left(\sigma \partial_{0}^{2}-\Delta_{3}\right)\right)^{j} E_{l, 1+j} \\
E_{l, 1+j}=\frac{Y\left(x_{0} / \sqrt{\sigma}-\left|x_{3}\right|\right)}{2 \sqrt{\sigma} \eta^{2+2 j} j !^{2}}\left(x_{0}^{2} / \sigma-x_{3}^{2}\right)^{j} \otimes \delta_{x_{1}} \otimes \delta_{x_{2}} .
\end{gathered}
$$


A more laborious computation yields $Z^{\mathrm{adj}}=Z_{1}^{\mathrm{adj}}+Z_{2}^{\mathrm{adj}}+Z_{3}^{\mathrm{adj}}$ with

$$
\begin{aligned}
& Z_{1}^{\text {adj }}(\partial)=\eta^{2}\left(\begin{array}{cccc}
\partial_{0} & 0 & 0 & -\partial_{3} \\
0 & 0 & 0 & 0 \\
0 & 0 & 0 & 0 \\
-\partial_{3} & 0 & 0 & \sigma \partial_{0}
\end{array}\right), \\
& Z_{2}^{\text {adj }}(\partial)=\eta\left(\begin{array}{cccc}
0 & -\partial_{0} \partial_{2} & \partial_{0} \partial_{1} & 0 \\
\partial_{0} \partial_{2} & 0 & \partial_{3}^{2}-\sigma \partial_{0}^{2} & -\partial_{2} \partial_{3} \\
-\partial_{0} \partial_{1} & \sigma \partial_{0}^{2}-\partial_{3}^{2} & 0 & \partial_{1} \partial_{3} \\
0 & \partial_{2} \partial_{3} & -\partial_{1} \partial_{3} & 0
\end{array}\right), \\
& Z_{3}^{\text {adj }}(\partial)=\partial_{0}\left(\begin{array}{ccccc}
\partial_{0}^{2} & -\partial_{0} \partial_{1} & -\partial_{0} \partial_{2} & -\partial_{0} \partial_{3} \\
-\partial_{0} \partial_{1} & \sigma \partial_{0}^{2}-\partial_{2}^{2}-\partial_{3}^{2} & \partial_{1} \partial_{2} & \partial_{1} \partial_{3} \\
-\partial_{0} \partial_{2} & \partial_{1} \partial_{2} & \sigma \partial_{0}^{2}-\partial_{1}^{2}-\partial_{3}^{2} & \partial_{2} \partial_{3} \\
-\partial_{0} \partial_{3} & \partial_{1} \partial_{3} & \partial_{2} \partial_{3} & \sigma \partial_{0}^{2}-\partial_{1}^{2}-\partial_{2}^{2}
\end{array}\right)
\end{aligned}
$$

Let us remark that $Z_{2}^{\mathrm{adj}}$ is skew-symmetric and $Z_{1}^{\mathrm{adj}}, Z_{3}^{\mathrm{adj}}$ are symmetric because of the identity $Z(\partial ;-\eta)=Z(\partial ; \eta)^{T}$. We finally conclude that $E_{z}=Z^{\text {adj }}(\partial) E$ has the following quaiasymptotic expansion:

$$
\begin{aligned}
E_{z}(k x) \sim \frac{k^{-s}}{2 \sqrt{\sigma} \eta^{2}} & Z_{1}^{\mathrm{sdj}}(\partial) Y\left(x_{0} / \sqrt{\sigma}-\left|x_{3}\right|\right) \otimes \delta_{x_{1}} \otimes \delta_{x_{2}}+\sum_{j=0}^{\infty} k^{-4-2 j} Z_{2}^{\mathrm{adj}}(\partial) T_{2 j} \\
& +\sum_{j=0}^{\infty} k^{-5-2 j}\left[Z_{1}^{\mathrm{sdj}}(\partial) \partial_{0}^{2}\left(\sigma \partial_{0}^{2}-\Delta_{3}\right) \frac{x_{3}^{2}-x_{0}^{2} / \sigma}{\eta^{2}(j+1)^{2}}+Z_{3}^{\mathrm{adj}}(\partial)\right] T_{2 j}
\end{aligned}
$$

In this example, $\operatorname{det} Z(0)=0$, and this fact renders the application of the next proposition impossible.

PRoposition 2. Let $Z(\partial)=\sum_{t_{0}}^{m} Z_{0}(\partial), Z_{0, i}$, homogeneous of degree $s$, and $E_{z}$ be as at the beginning of the section (i. e., $\operatorname{det} Z(-i z) \neq 0$ in $T^{C}$ ) and assume that $\operatorname{det} Z_{0}=\operatorname{det} Z(0) \neq 0$. Then the quasi-asymptotic expansion of $E_{Z}(k x)$ in $S^{\prime}(\Gamma)^{N \times N}$ for $k \rightarrow \infty$ is given by the formula

$$
E_{z}(k x) \sim \sum_{r=0}^{\infty} k^{-n-r} \sum_{\substack{r_{1}+\cdots+r_{j}=r \\ j \geq 0,1 \leq r_{i} \leq m}}(-1)^{j} Z_{0}^{-1} Z_{r_{1}}(\partial) Z_{0}^{-1} \ldots Z_{0}^{-1} Z_{r_{j}}(\partial) Z_{0}^{-1} \delta
$$

PROOF: As in the proof of Proposition 1, the quasi-asymptotic expansion in question is transformed to one in $H(C)$. Because of the hypothesis det $Z(0) \neq 0$, the matrixvalued function $Z(-i z t)^{-1}$ is analytic in $t=0$ for fixed $z \in T^{C}$ and its Taylor series 
is given by

$$
\begin{aligned}
Z(-i z t)^{-1} & =\left[Z_{0}\left(I+\sum_{r=1}^{m} Z_{0}^{-1} Z_{r}(-i z) t^{r}\right)\right]^{-1} \\
& =\sum_{j=0}^{\infty}\left(-\sum_{r=1}^{m} Z_{0}^{-1} Z_{r}(-i z) t^{r}\right)^{j} Z_{0}^{-1} \\
& =\sum_{r=0}^{\infty} t^{r} \sum_{\substack{1_{1}+\cdots+r_{j}=r \\
j \geq 0,1 \leq r_{i} \leq m}}(-1)^{j}\left(Z_{0}^{-1} Z_{r_{1}} Z_{0}^{-1} \ldots Z_{0}^{-1} Z_{r}, Z_{0}^{-1}\right)(-i z)
\end{aligned}
$$

for $|t|<\epsilon(z)$. Herein $I$ denotes the unit matrix. The rest of the proof proceeds in a similar manner as that of Proposition 1 . In fact, the integral remainder term now contains derivatives with respect to $s$ of

$$
Z(-i z s)^{-1}=P(-i z s)^{-1} Z^{\mathrm{sd} i}(-i z s)
$$

wherein $P=\operatorname{det} Z$ fulfils the conditions presumed in Proposition 1. Therefore, the same procedure works, and hence the proof is complete.

EXAMPLE 4 (cf. $[9, \S 12.5]$ ). Let us consider the Dirac-operator

$$
Z=\sum_{\mu=0}^{3} i \gamma^{\mu} \partial_{\mu}-M=i \not \nabla-M
$$

where, for convenience, we use the so-called Feynman dagger (cf. [1]). The causal fundamental solution is

$$
\begin{gathered}
E_{z}=-(i \notin+M)\left[\frac{1}{4 \pi} \delta\left(x_{0}-|\mathbf{x}|\right)-\frac{M}{4 \pi} Y\left(x_{0}-|\mathbf{x}|\right) \frac{J_{1}\left(M \sqrt{x^{2}}\right)}{\sqrt{x^{2}}}\right] \\
\mathbf{x}=\left(x_{1}, x_{2}, x_{3}\right), x^{2}=x_{0}^{2}-x_{1}^{2}-x_{2}^{2}-x_{3}^{2}
\end{gathered}
$$

(see $[\boldsymbol{T}, \S 11.12\})$. Proposition 2 yields the following quasi-asymptotic expansion:

$$
\begin{aligned}
E_{Z}(k x) \sim & -\frac{1}{M} \sum_{r=0}^{\infty} k^{-4-r}\left(\frac{i}{M} \nabla\right)^{r} \delta \\
& =\sum_{j=0}^{\infty} k^{-4-2 j} \frac{(-1)^{j+1}}{M^{2 j+1}} I \square^{j} \delta+\sum_{j=0}^{\infty} k^{-\delta-2 j} \frac{i^{2 j+3}}{M^{2 j+2}} \nabla \square^{j} \delta
\end{aligned}
$$




\section{REFERENCES}

[1] FEYNMAN, R.P.: Quantum Electrodymamics. New York: Benjamin 1961.

(2) HORMANDER, L.: On the division of distributions by polynomials. Ark. Mat. 3 (1958), 555 568

[3] HÖRMANDER,L.: Linear Partial Differential Operators, 3 nd ed. (Crundlehren der Mathe matischen Wissenschaften: Vol. 116). Berlin: Springer-Verlag 1969.

[4] HÖMANDER, L.: The Analysis of Linear Partial Differential Operators, Vol. 11 (Grundlehren der Mathematischen Wissenschaften: Vol. 257). Berlin: Springer-Verlag 1983.

[5] ORTNER, N.: Regularisjerte Faltung von Distributionen. ZAMP 31 (1980), 133 - 173.

[6] SCHWARTZ. L:: Theorie des Distributions. Nouvelle édition. Paris: Hermann 1966.

[7] VLADIMIROV, V.S.: Equations of Mathematical Physics, 5 th ed. (in Russian). Moscow: Nauka 1988. English translation: New York: Decker 1971.

[8] VLADIMIROV,V.S.: Generalized Functions in Mathematical Physics, 2nd ed. (in Russian). Moscow: Nauka 1979. English translation: Moscow: Mir 1979.

[9] VladimiRoV, V.S., DroZhzHINOV, YU.N., and B. I. ZAV'YALOV.: Multidimensional Tauberian Theorems for Generalized Functions (in Russian). Moscow: Nauka 1986. English translation: Dortrecht: Reidel 1988.

[10] WAGNER, P.: Bernstein-Sato-Polynome und Faltungsgruppen zu Differentialoperatoren. Z. Anal. Anw. 8 (1989), $407-423$.

Received 18.12.1989

Author's address:

Dr. Peter Wagner

Institut für Mathematik und Geometrie

Universitat Innsbruck

A-6020 Innsbruck

\section{Book reviews}

O. GREUEL and H. KADNER: Komplexe Funktionen und konforme Abbildungen (Mathematik für Ingenieure, Naturwissenschaftler, Ökonomen und Landwirte: Vol. 9). Leipzig:B.G. Teubner Verlagsges. 1990, 128 pp., 116 fig.

The book is a well-written introduction into Complex Analysis, especially for those studying Mathematics as subsidiary. It meets, therefore, the needs of the series "Mathematics for engineers, scientists, economists, and farmers" the book belongs to. In accordance with the interests of such readers the authors prefer explicit calculations (instead of long and abstract proofs) and applications to other fields of Mathematical Analysis such as. the calculation of real integrals. Some theorems (such as Riemann's mapping theorem) are formulated without proofs, others are proved by clear arguments partly showing interac-tions between Real and Complex Analysis (e.g. the Cauchy integral theorem is proved by applying properties of real line integrals). That way the book contains many references to other books of the abovementioned series, too. According to its title the book also investigates many (elementary) conformal mappings. The book containing a lot of exer-cises (whose solutions take 12 pages) is worthy of recommendation.

Halle

W. TUTSCHKE 